\title{
Radiation Segmentectomy
}

\author{
Kristina Prachanronarong, $\mathrm{MD}, \mathrm{PhD}^{1} \quad$ Edward Kim, $\mathrm{MD}^{1}$ \\ ${ }^{1}$ Department of Interventional Radiology, Icahn School of Medicine at \\ Mount Sinai, Mount Sinai Health System, New York, New York \\ Semin Intervent Radiol 2021;38:425-431 \\ Address for correspondence Edward Kim, MD, FSIR, Division of \\ Radiology and Surgery, Department of Interventional Oncology, \\ Mount Sinai Health System, New York, NY 10029 \\ (e-mail: Edward.Kim@mountsinai.org).
}

\author{
Abstract \\ Keywords \\ - radioembolization \\ - radiation \\ segmentectomy \\ - hepatocellular \\ carcinoma \\ - interventional \\ radiology
}

Radiation segmentectomy is an yttrium-90 transarterial radioembolization treatment where a high radiation dose is administered to a small volume of liver to achieve a high tumoricidal dose to a target with anatomic surgical precision while sparing surrounding parenchyma. This therapeutic modality is often used to treat hepatocellular carcinoma, and recent studies have demonstrated that radiation segmentectomy is an effective treatment as a neoadjuvant to transplant, resection, or as a standalone treatment. This article provides a review of radiation segmentectomy, indications for treatment, recent outcome data, and guidelines for postprocedural management.
The development of locoregional therapies such as yttrium90 (Y90) transarterial radioembolization has led to interventions that are analogous in anatomic precision to surgery but are minimally invasive, increasing patient eligibility for these treatments, improving treatment of lesions in complex anatomic locations, and maximizing effectiveness while reducing side effects. ${ }^{1,2}$ Radiation segmentectomy is a Y90 transarterial radioembolization treatment where a high radiation dose is administered to a small volume of liver to achieve a high tumoricidal dose to a target with anatomic surgical precision while sparing surrounding parenchyma., ${ }^{1,3}$ Particles embedded with radioactive isotopes are delivered through hepatic arteries to a target tumor where the particles seed the tumor tissue and emit radiation over a period of time to treat the target lesion. This treatment is based on an important principle of transarterial liver tumor treatment that liver tumors receive a majority of their blood supply from the hepatic arterial system, while normal liver parenchyma is supplied by the portal venous system. Radiation segmentectomy is described as radioembolization during a single-treatment session of two or fewer hepatic segments, defined by the Couinaud system. ${ }^{4,5}$ Radiation segmentectomy results in ablation of the treated lesion and atrophy of the perfused segment on follow-up imaging. High radiation doses are delivered to the target lesion, leading to improved treatment response and a high objective response rate (ORR) which have been validated by several studies, most recently culminating in the LEGACY study, which evaluated Local
radioEmbolization using glass microspheres for the Assessment of Tumor Control with Y90. ${ }^{6}$

Radiation segmentectomy was initially developed in 2011 in response to clinical need for selective treatment of liver lesions in patients who were not good candidates for other treatments such as thermal ablation or surgery based on considerations such as anatomy, comorbidities, and functional liver reserve. The treatment was designed to deliver high radiation activity directly to a target lesion while sparing surrounding tissue. ${ }^{5,7,8}$ Lower radiation doses applied to surrounding normal parenchyma have been suggested to reduce injury and promote improved regeneration of normal tissue after treatment. ${ }^{5}$ Radiation segmentectomy has undergone an iterative process of research, clinical trials, and development eventually culminating in the LEGACY study which was recently published in March 2021. The LEGACY study demonstrated that Y90 radioembolization and radiation segmentectomy are effective treatments as neoadjuvant to transplant, resection, or as a standalone treatment for lesions up to $8 \mathrm{~cm}$, supporting the use of a perfused volume absorbed dose of greater than $400 \mathrm{~Gy}$ as a "threshold" dose for an ablative effect with an $88 \%$ ORR over 24 months. ${ }^{6}$

\section{Indications for Treatment}

Radioembolization treatment is indicated for patients with unresectable hepatocellular carcinoma (HCC), cholangiocarcinoma, or metastatic liver lesions. For patients with HCC,
Issue Theme Seminars in Radioembolization; Guest Editors, Robert J. Lewandowski, MD, FSIR and William Rilling, MD (c) 2021. Thieme. All rights reserved. Thieme Medical Publishers, Inc., 333 Seventh Avenue, 18th Floor, New York, NY 10001, USA
DOI https://doi.org/ 10.1055/s-0041-1735529. ISSN 0739-9529. 
radioembolization applications range from very early stage HCC to advanced stage HCC based on the modified Barcelona Clinic Liver Cancer (BCLC) staging system and treatment strategy recommendations from the European Association for the Study of Liver (EASL) and the American Association for the Study of Liver Diseases (AASLD). ${ }^{9}$ Radiation segmentectomy can be used for curative intent as a standalone treatment or neoadjuvant to transplant or resection. Patients with small tumors $(\leq 3 \mathrm{~cm})$ are generally considered for curative treatments such as transplantation, thermal ablation, and surgical resection. ${ }^{10}$ Surgical resection is curative for patients with solitary tumor, normal bilirubin, and absence of portal hypertension; however, patients often do not meet these criteria. ${ }^{9}$ Thermal ablation is also sometimes limited due to target lesion size (generally $<3 \mathrm{~cm}$ ) and high-risk location. More recently, the LEGACY study has shown that radiation segmentectomy is potentially a curative treatment option. ${ }^{6}$ If a target lesion is not amenable to more invasive treatment such as thermal ablation or surgical resection due to anatomic location (e.g., in close proximity to large vessels or adjacent to the diaphragm or dome of the liver), patient comorbidities, or limited functional liver reserve, radiation segmentectomy should be considered.

Bridging as well as downstaging patients for transplant is an important indication for treatment with radiation segmentectomy. The LEGACY study showed that treatment with Y90 microspheres for solitary unresectable HCC allowed $85.3 \%$ of patients to attain and/or maintain Milan criteria after treatment at 24 months, which is significant due to often prolonged wait times for transplant, which can vary based on factors such as region and donor/recipient blood type. ${ }^{6}$ Radioembolization can be used to "bridge" a patient to transplantation by controlling tumor burden prior to transplant and to "downstage" a patient to become eligible for transplant by controlling tumor burden to fit specific transplant eligibility tumor criteria. ${ }^{11}$ The management of HCC is guided by the BCLC algorithm which provides treatment recommendations based on staging determined by performance status, underlying liver disease, and tumor burden extent. $^{10-12}$ Liver transplantation is a curative therapy for HCC as part of the BCLC algorithm, but for patients to qualify for liver transplant, they must fit "Milan" criteria, which is a set of criteria developed at the National Cancer Institute in Milan, Italy, showing survival benefit for patients with HCC tumor burden meeting specific criteria, such as single tumors $\leq 5 \mathrm{~cm}$ in diameter or three or fewer tumors $\leq 3 \mathrm{~cm}$ in diameter without vascular invasion or metastases. ${ }^{11,13,14}$ In addition, they must undergo a 6-month waiting period related to their calculated Model of End-Stage Liver Disease (MELD) score before receiving exception points for HCC. ${ }^{15}$ Radiation segmentectomy is beginning to be recognized as a curative therapy, is often better tolerated than other locoregional therapies such as transarterial chemoembolization (TACE) without significant differences in downstaging/bridging success rates, and offers additional advantages discussed in more detail below.

Radiation segmentectomy has specific advantages and disadvantages compared with other treatments such as thermal ablation, external-beam radiation therapy (EBRT), and transarterial chemoembolization. Radiation segmentec- tomy uses particles with increased specific radiation activity to deliver a high treatment dose directly to a target while sparing surrounding parenchyma, which is an important advantage over treatments such as EBRT where a larger proportion of normal parenchyma is impacted in the process of treating the target lesion. With radiation segmentectomy, there is no radiation dose limit compared with EBRT. Advantages of radioembolization versus EBRT are that certain lesions are easier to access via radioembolization, such as targets within the caudate lobe and the dome of the liver, where treatment with EBRT would risk damage to surrounding structures such as the lungs and porta hepatis. ${ }^{5}$ In addition, treatment with EBRT often includes multiple sessions, and respiratory motion can expose lung parenchyma to possible radiation damage. 5,16

Some advantages of radioembolization versus thermal ablation are that percutaneous needle and probe placement are avoided, minimizing the theoretical risk of tract seeding, and high-risk ablation lesions (based on anatomic location or patient comorbidities) are often more easily targeted with radiation segmentectomy. ${ }^{5,8}$ Another advantage of radioembolization is that while thermal ablation as a curative intent therapy has been validated with studies for lesions less than $3 \mathrm{~cm}$ for HCC, several groups including Salem et al and Kim et al have demonstrated favorable response rates for lesions up to $8 \mathrm{~cm}$ in size using radiation segmentectomy. ${ }^{6,17-20}$ Some examples of high-risk lesions include those located in the caudate lobe, near the diaphragm and lung tissue, large vessels and biliary structures, or located near other organs such as the heart, gallbladder, or small or large bowel. ${ }^{5}$ Disadvantages of radioembolization versus ablation are that there is potentially increased cost and reliance on hypervascularity to the tumor, as some tumors are hypovascular. ${ }^{8}$

Radioembolization also has specific advantages compared with TACE in that the particles used for radioembolization are ideally sized for minimal stasis and to permit continued blood flow through the hepatic arteries after treatment, which potentiates the effects of radiation and also maintains access to the tumor lesion if other additional treatments subsequently need to be delivered to the target tissue. With TACE, a chemotherapeutic agent is injected into the target lesion and the target vessels are occluded to destroy the lesion. Another advantage is that radiation segmentectomy has been shown to be safe and effective for patients with moderate hepatic dysfunction and advanced disease. ${ }^{21}$ In 2017, Padia et al reported that radiation segmentectomy has greater complete response rates and local tumor control compared with TACE with similar side effect profiles. ${ }^{22}$ Biederman et al also showed similar outcomes in patients treated with radiation segmentectomy for HCC up to $3 \mathrm{~cm}$ compared with TACE and microwave ablation, with improved imaging response and increased time to secondary treatment compared with TACE. ${ }^{23,24}$

\section{Radiation Segmentectomy Outcome Data}

The LEGACY study is a retrospective, single-arm, multicenter study conducted at three U.S. sites (Northwestern 
University in Chicago, IL; University of Washington, Seattle, WA; and Mount Sinai Health System, New York, NY) that evaluated consecutive patients treated with Y-90 glass microspheres between January 2014 and December 2017 with median follow-up of 29.9 months (95\% confidence interval [CI]: $24.7-34.6){ }^{6}$ The purpose of the study was to assess patient outcomes when glass microspheres are used to treat early or advanced HCC by evaluating a primary endpoint of local tumor control ORR and duration of response (DOR) following treatment with Y90 glass microspheres in unresectable solitary HCC lesions. These endpoints are similar endpoints that have been published for phase III randomized controlled clinical trials of systemic therapies for the treatment of advanced-stage HCC. The patient population age ranges from 18 to greater than 75 years with approximately $18 \%$ of the population being older than 75 years. Eastern Cooperative Oncology Group (ECOG) status and BCLC status ranged from 0 to 1 and A to C, respectively. Median tumor size was $2.6 \mathrm{~cm}$ with a range from 0.9 to $8.1 \mathrm{~cm}$, and the absorbed dose to perfused liver volume median was $410.1 \mathrm{~Gy}$ (interquartile range: 199.7797.7). This dose threshold produced a high treatment response rate, and using a localized mRECIST (modified Response Evaluation Criteria in Solid Tumors) within the radioembolization-treated region at 24 months, $100 \%$ of patients were without localized tumor progression and $94 \%$ of patients had progression-free survival. Local recurrence rate for the study was approximately $5.6 \%$ and ORR was $88.3 \%$. At 24 months, $85 \%$ of patients were able to maintain or attain the Milan criteria for transplantation, and $82 \%$ maintained or improved baseline ECOG status. Overall, the LEGACY study demonstrated that Y90 radioembolization is an effective treatment as neoadjuvant to transplant, resection, or as a standalone treatment, and supports the use of a perfused volume-absorbed dose of greater than $400 \mathrm{~Gy}$.

The LEGACY study and selected additional radiation segmentectomy studies are listed in - Table 1 . These studies evaluated imaging response and overall survival after radiation segmentectomy for HCC with Y90 glass microspheres using various criteria to evaluate imaging response to treatment, including guidelines from the EASL, World Health Organization (WHO), and mRECIST. Of note, in a review of these studies, median time-to-disease progression ranged from 7.9 to 33.3 months. ${ }^{3,23-27}$ Biederman et al compared outcomes of radiation segmentectomy and TACE in the treatment of unresectable solitary HCC less than or equal to $3 \mathrm{~cm}$ in size and found improved efficacy of radiation segmentectomy in a homogeneous cohort with respect to HCC stage. ${ }^{24}$ Using mRECIST criteria, they reported complete response in $81.2 \%$ of patients after radiation segmentectomy and after propensity score matching, complete response was $92.1 \%{ }^{24}$ The same group also evaluated radiation segmentectomy versus TACE combined with microwave ablation for unresectable solitary $\mathrm{HCC}$ up to $3 \mathrm{~cm}$ and showed that imaging response and progression outcomes were not significantly different between the two groups with complete response of $82.9 \%$ for radiation segmentectomy versus $82.5 \%$ for TACE with microwave ablation. ${ }^{23}$
Lewandowski et al evaluated long-term outcomes ( $>10$ years) of radiation segmentectomy for the treatment of early HCC and showed that response rates, tumor control, and survival outcomes are comparable to treatments such as ablation for patients with BCLC stage 0 or A HCC lesions. ${ }^{3} \mathrm{Kim}$ et al addressed the use of a boosted dose of Y90 to large HCC lesions greater than $5 \mathrm{~cm}$ in size and showed favorable tumor response but with increased biliary complications such as biliary strictures. ${ }^{20}$ In an earlier study, Vouche et al used mRECIST criteria to evaluate treatment response on imaging and showed objective response in $88 \%$ and stable disease in $12 \%$ of patients. ${ }^{8}$ Median time-to-disease progression in this trial was 33.1 months, with a majority of cases with disease progression secondary to new intrahepatic lesions and not the previously treated lesion. In the earliest experience, Riaz et al used EASL and WHO criteria to evaluate treatment response on imaging and demonstrated EASL response in $81 \%$ of patients with median time to response of 1.2 months and WHO response in $59 \%$ of patients with median time to response of 7.2 months. $^{5}$

Overall median survival rates with radiation segmentectomy range from 13.6 to 80.4 months. ${ }^{3,5,8,24}$ Variability in overall survival is likely related to several factors including study duration, advances in treatment technique, and availability of new medical treatments while studies are in progress. For instance, the duration of the largest studies ranges between 5 and 8 years, and advances in treatment technique and use of specific medical treatments during some of these studies became more common, such as the use of sorafenib when it was approved in 2007.

The objective of radiation segmentectomy is complete pathological necrosis (CPN) of the target lesion analogous to what is seen with ablation, and several studies have focused on the radiation dose necessary to achieve this goal. ${ }^{28}$ In particular, the LEGACY study has established that a perfused liver treatment dose greater than $400 \mathrm{~Gy}$ is the dose threshold for a high ORR, achieving more than $96 \%$ response rate in patients with lesions less than $3 \mathrm{~cm}$ in size. ${ }^{6}$ Although there are limitations comparing radiation segmentectomy to other types of therapies such as percutaneous ablation, studies suggest that overall survival and localized tumor response do not differ significantly between radiation segmentectomy and ablation when patients are stratified based on level of liver dysfunction (e.g., Childs-Pugh score).$^{8,17-19,29}$ Furthermore, radiation segmentectomy offers advantages where ablation is limited, such as with complex lesions in highrisk anatomic locations. ${ }^{30,31}$

\section{Postprocedural Complications and Toxicities}

Postprocedural complications after radiation segmentectomy are similar to those that have been described for other Y90 radioembolization procedures. For instance, some symptoms that can be expected after radiation segmentectomy include fatigue, fevers, and chills, which may be due to the release of endogenous pyrogens secondary to the effect of radiation on normal hepatic tissue. ${ }^{32-34}$ Additional possible clinical adverse events include pain, nausea, vomiting, 


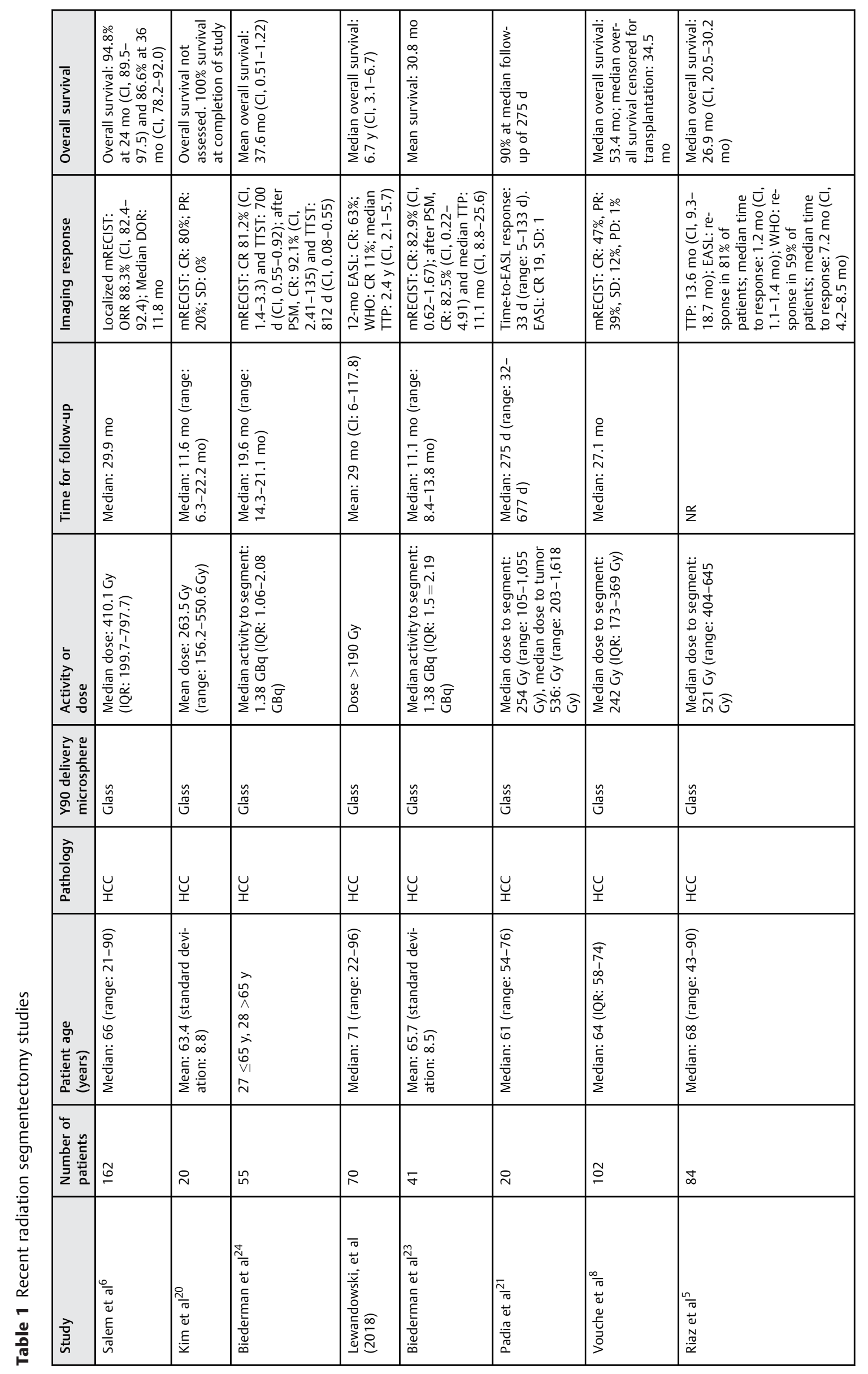

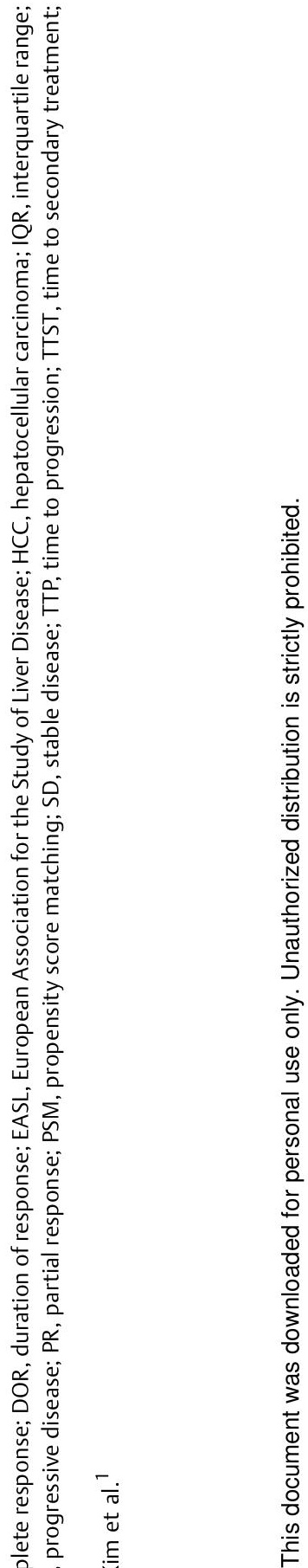


diarrhea, anorexia, and weight loss. ${ }^{5,8}$ Laboratory abnormalities that can be expected after radiation segmentectomy include elevations in measures of liver function such as bilirubin, albumin, international normalized ratio, platelet levels, aspartate aminotransferase, alanine transaminase, alkaline phosphatase, and lymphocyte counts. However, delineating toxicities from radiation segmentectomy versus preexisting liver dysfunction or progression of liver dysfunction due to underlying disease is difficult. ${ }^{4,8}$

Another potential risk of $Y 90$ radioembolization procedures is radioembolization-induced liver disease (REILD) which is comparable to radiation-induced liver disease (RILD) and which encompasses symptoms such as fatigue, jaundice, and ascites that occur 1 to 2 months after treatment with external beam radiation therapy without subsequent bile obstruction or tumor progression. ${ }^{35,36}$ These syndromes are a subset of venoocclusive disease (VOD). REILD presents with markedly increased bilirubin in contrast to RILD, which is characterized by relatively proportional liver enzyme elevation. ${ }^{36}$ Factors such as worsening liver function, elevated bilirubin, cirrhosis, prior treatment with chemotherapy, young age, volume of treated liver, and ratio of activity administered to treated liver volume are risks for the development of REILD. ${ }^{36-38}$ With radiation segmentectomy, the volume of normal liver parenchyma that is affected is reduced without impacting treatment efficacy, and consequently, REILD is less of a risk. ${ }^{36}$ Notably, during the LEGACY study, none of the patients experienced REILD. ${ }^{6}$ Because the radiation dose is targeted to one to two segments of the liver which is a smaller volume of liver parenchyma, there is a theoretical risk of bilirubin toxicity and biliary complications. ${ }^{4,5}$ In one study of 84 patients who underwent radiation segmentectomy, $5 \%$ of patients developed small postprocedural bilomas within the treated segment. ${ }^{5}$ Kim et al reported biliary strictures in some patients who underwent boosted dose Y90 for large HCC lesions greater than $5 \mathrm{~cm}$ in size. ${ }^{20}$ However, in additional studies such as a multicenter study of 102 patients, a separate study of 20 patients, and the LEGACY study, there were no reports of bilomas. ${ }^{6,8,21}$ This discrepancy may be due to variability in techniques, such as superselectivity, which may theoretically increase the risk of biloma. There were some cases of hepatobiliary disorders seen in the LEGACY study, where a small number of patients experienced posttreatment gallbladder obstruction $(N=1)$, portal vein thrombosis $(N=1)$, ascites $(N=3)$, and increase in bilirubin $(N=3) .^{6}$ In contrast, with lobar radiation treatments, these types of focal bilirubin complications or toxicity are more common. ${ }^{4,39}$

\section{Postprocedural Patient Management}

\section{Immediate Postprocedural Care}

Radiation segmentectomy is an outpatient procedure, and patients recover for approximately 2 to 6 hours postprocedurally before discharge home. In general, patients are discharged home with pain medication, gastrointestinal ulcer prophylaxis such as proton pump inhibitors, antiemetics, and, rarely, steroid tapers for treatment of fatigue, similar to

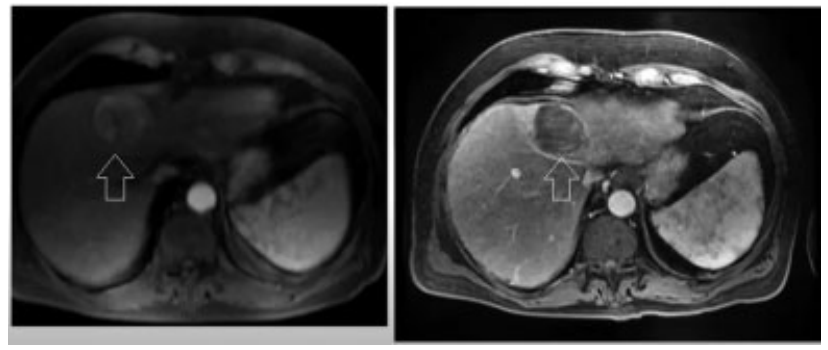

Fig. 1 Magnetic resonance T1-weighted gadolinium-enhanced arterial phase sequence prior to radiation segmentectomy (a) demonstrates a segment 4 lesion (arrows) measuring $5.0 \mathrm{~cm}$. (b) 2 years post radiation segmentectomy demonstrates complete response as per mRECIST.

usual postprocedural radioembolization care. Prior to discharge, patients should be reminded of possible often selflimited adverse events they may experience, such as fevers, chills, pain, nausea, and vomiting and radiation safety precautions if applicable. ${ }^{34}$

\section{Outpatient Follow-up Evaluation}

Patients are usually seen for follow-up in an outpatient clinic approximately 4 to 6 weeks after treatment. While the majority of patients will have few side effects, clinicians should evaluate patients for changes in performance status as well as monitoring for adverse effects, such as tumor lysis syndrome or nontarget organ toxicity such as adverse events within the lungs or gastrointestinal system. Many of the potential immediate postprocedural adverse clinical events described previously are self-limited; however, further evaluation should be considered if symptoms persist. Follow-up laboratory tests may show transient increases in liver function tests and tumor markers and decreases in lymphocytes. ${ }^{34}$ Follow-up imaging is also recommended with contrast-enhanced CT or MRI approximately 1 to 3 months posttreatment and then at 3- to 6-month intervals to evaluate treatment response and guide future treatment planning. Median time to objective response per mRECIST has been demonstrated at 1.2 and 6.6 months for change in size as per mRECIST. ${ }^{26,40-42}$

\section{Clinical Cases with Images}

In one representative case of radiation segmentectomy for the treatment of HCC, a patient with a past medical history of solitary unresectable $\mathrm{HCC}$ measuring $5 \mathrm{~cm}$ in segment 4 was treated with Y90 radiation segmentectomy, and on MRI follow-up 2 years later, the patient had complete response based on mRECIST with unchanged liver function and with AFP dropping to within normal limits from 600 . This patient underwent liver transplant 5 years after treatment and explant pathology showed complete necrosis without tumor recurrence (-Fig. 1). In another case, a patient with a segment 4 HCC was treated with Y90 radiation segmentectomy, and on follow-up, the patient had complete treatment response and remained free of disease progression 21 months after treatment ( - Fig. 2). These cases provide examples of how radiation segmentectomy is a potentially 


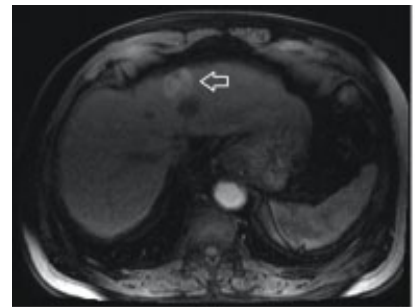

(a)

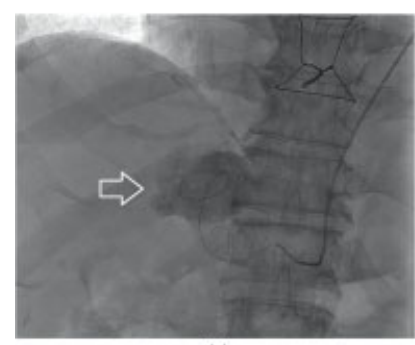

(c)

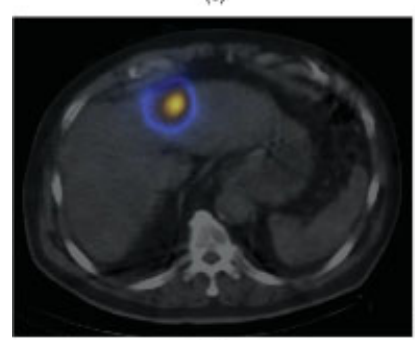

(e)

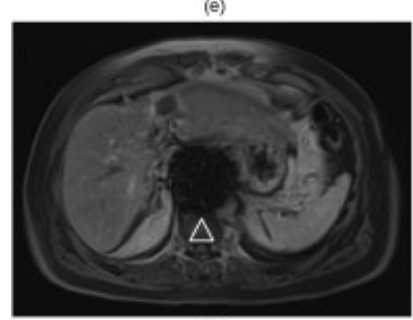

(g)

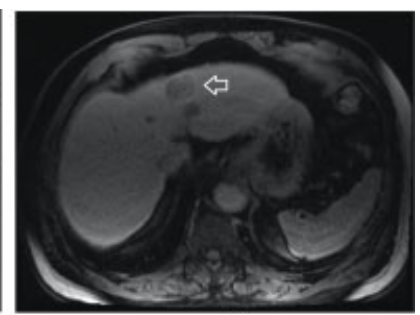

(b)

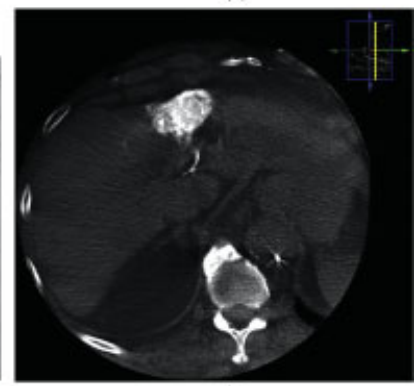

(d)

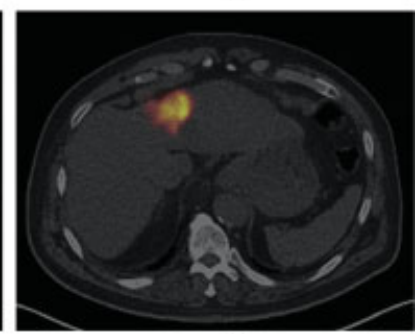

(f)

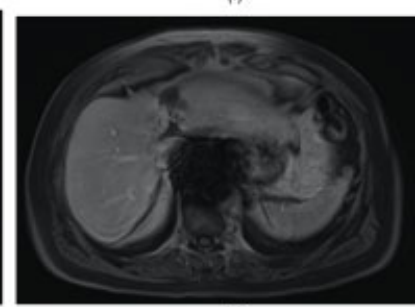

(h)

Fig. 2 Arterial (a) and venous (b) phase axial T1-weighted postcontrast images demonstrate a segment $4 \mathrm{HCC}$ (arrows) with early arterial enhancement and portal venous washout. During angiography (c), sub-selection of the segment 4 hepatic artery shows tumor blush (arrow) correlating with the tumor seen on MRI. An arterial contrast injection using cone-beam CT imaging (d) demonstrates good coverage of the HCC and adjacent parenchyma with radiation segmentectomy. Immediately after radioembolization, a fused bremsstrahlung and CT image (e) show that the delivered radiation dose is concentrated within the tumor which is confirmed by PET/CT obtained following treatment (f). Posttreatment evaluation with axial T1-weighted MRI shows no enhancement in the tumor bed on arterial (g) and venous (h) phases, consistent with complete treatment response. Artifact from an interval abdominal aortic aneurysm repair is incidentally seen (arrowhead). (Reprinted with permission from Titano J, Voutsinas N, Kim E. The role of radioembolization in bridging and downstaging hepatocellular carcinoma to curative therapy. Semin Nucl Med 2019;49(3):189-196. ${ }^{11}$ )

curative treatment modality that is well tolerated and has superior tumor response. The second case also illustrates an example of how cone beam CT can be used to evaluate intraprocedural tumor treatment volume to provide up-todate volume measurements for dose calculations prior to treatment. $^{43}$

\section{Conclusions}

Radiation segmentectomy is an important treatment option for patients with a spectrum of disease, ranging from very early stage to early-stage patients with target lesions in anatomically challenging locations to patients with more intermediate to advanced disease who may benefit from downstaging treatment to become eligible for liver transplantation, and patients with advanced stage disease with portal invasion and extrahepatic spread. Understanding factors such as indications for treatment, patient selection, dosimetry, tumor biology, postprocedural care, and expected patient outcomes is critical for the appropriate application of radiation segmentectomy in patient care.

\section{Conflict of Interest}

K.P. has no conflict of interest. E.K. is on the scientific advisory board for Boston Scientific Corporation and Sirtex Medical.

\section{References}

1 Kim E, Titano JJ, Lekperic S. Radioembolization in Segmentectomy, Lobectomy, and Future Liver Remnant Hypertrophy. CRC Press; 2017:131-150

2 Titano J, Noor A, Kim E. Transarterial chemoembolization and radioembolization across Barcelona Clinic Liver Cancer Stages. Semin Intervent Radiol 2017;34(02):109-115

3 Lewandowski RJ, Gabr A, Abouchaleh N, et al. Radiation segmentectomy: potential curative therapy for early hepatocellular carcinoma. Radiology 2018;287(03):1050-1058

4 Rhee TK, Omary RA, Gates V, et al. The effect of catheter-directed CT angiography on yttrium-90 radioembolization treatment of hepatocellular carcinoma. J Vasc Interv Radiol 2005;16(08): 1085-1091

5 Riaz A, Gates VL, Atassi B, et al. Radiation segmentectomy: a novel approach to increase safety and efficacy of radioembolization. Int J Radiat Oncol Biol Phys 2011;79(01):163-171

6 Salem R, Johnson GE, Kim E, et al. Yttrium-90 radioembolization for the treatment of solitary, unresectable hepatocellular carcinoma: the LEGACY study. Hepatology 2021 (epub ahead of print). Doi: 10.1002/hep.31819

7 Ben-Josef E, Normolle D, Ensminger WD, et al. Phase II trial of high-dose conformal radiation therapy with concurrent hepatic artery floxuridine for unresectable intrahepatic malignancies. J Clin Oncol 2005;23(34):8739-8747

8 Vouche M, Habib A, Ward TJ, et al. Unresectable solitary hepatocellular carcinoma not amenable to radiofrequency ablation: multicenter radiology-pathology correlation and survival of radiation segmentectomy. Hepatology 2014;60(01):192-201

9 European Association for the Study of the Liver. Electronic address: easloffice@easloffice.eu European Association for the Study of the Liver. EASL Clinical Practice Guidelines: management of hepatocellular carcinoma. J Hepatol 2018;69(01): $182-236$

10 Llovet JM, Brú C, Bruix J. Prognosis of hepatocellular carcinoma: the BCLC staging classification. Semin Liver Dis 1999;19(03): 329-338

11 Titano J, Voutsinas N, Kim E. The role of radioembolization in bridging and downstaging hepatocellular carcinoma to curative therapy. Semin Nucl Med 2019;49(03):189-196

12 Forner A, Reig ME, de Lope CR, Bruix J. Current strategy for staging and treatment: the BCLC update and future prospects. Semin Liver Dis 2010;30(01):61-74 
13 Mazzaferro V, Regalia E, Doci R, et al. Liver transplantation for the treatment of small hepatocellular carcinomas in patients with cirrhosis. N Engl J Med 1996;334(11):693-699

14 Wald C, Russo MW, Heimbach JK, Hussain HK, Pomfret EA, Bruix J. New OPTN/UNOS policy for liver transplant allocation: standardization of liver imaging, diagnosis, classification, and reporting of hepatocellular carcinoma. Radiology 2013;266(02):376-382

15 Kulik L. Criteria for liver transplantation in hepatocellular carcinoma. Clin Liver Dis (Hoboken) 2015;6(04):100-102

16 Salem R, Thurston KG. Radioembolization with yttrium-90 microspheres: a state-of-the-art brachytherapy treatment for primary and secondary liver malignancies: part 3: comprehensive literature review and future direction. J Vasc Interv Radiol 2006;17 (10):1571-1593

17 Chen MH, Wei Y, Yan K, et al. Treatment strategy to optimize radiofrequency ablation for liver malignancies. J Vasc Interv Radiol 2006;17(04):671-683

18 Livraghi T, Meloni F, Di Stasi M, et al. Sustained complete response and complications rates after radiofrequency ablation of very early hepatocellular carcinoma in cirrhosis: Is resection still the treatment of choice? Hepatology 2008;47(01):82-89

19 Pompili M, Saviano A, de Matthaeis N, et al. Long-term effectiveness of resection and radiofrequency ablation for single hepatocellular carcinoma $\leq 3 \mathrm{~cm}$. Results of a multicenter Italian survey. J Hepatol 2013;59(01):89-97

20 Kim HC, Kim YJ, Lee JH, Suh KS, Chung JW. Feasibility of boosted radioembolization for hepatocellular carcinoma larger than $5 \mathrm{~cm}$. J Vasc Interv Radiol 2019;30(01):1-8

21 Padia SA, Kwan SW, Roudsari B, Monsky WL, Coveler A, Harris WP. Superselective yttrium-90 radioembolization for hepatocellular carcinoma yields high response rates with minimal toxicity. J Vasc Interv Radiol 2014;25(07):1067-1073

22 Padia SA, Johnson GE, Horton KJ, et al. Segmental yttrium-90 radioembolization versus segmental chemoembolization for localized hepatocellular carcinoma: results of a single-center, retrospective, propensity score-matched study. J Vasc Interv Radiol 2017;28(06):777-785.e1

23 Biederman DM, Titano JJ, Bishay VL, et al. Radiation segmentectomy versus TACE combined with microwave ablation for unresectable solitary hepatocellular carcinoma up to $3 \mathrm{~cm}$ : a propensity score matching study. Radiology 2017;283(03): 895-905

24 Biederman DM, Titano JJ, Korff RA, et al. Radiation segmentectomy versus selective chemoembolization in the treatment of early-stage hepatocellular carcinoma. J Vasc Interv Radiol 2018; 29(01):30-37.e2

25 Lewandowski RJ, Kulik LM, Riaz A, et al. A comparative analysis of transarterial downstaging for hepatocellular carcinoma: chemoembolization versus radioembolization. Am J Transplant 2009;9 (08):1920-1928

26 Salem R, Lewandowski RJ, Mulcahy MF, et al. Radioembolization for hepatocellular carcinoma using Yttrium-90 microspheres: a comprehensive report of long-term outcomes. Gastroenterology 2010;138(01):52-64

27 Sangro B, Salem R, Kennedy A, Coldwell D, Wasan H. Radioembolization for hepatocellular carcinoma: a review of the evidence and treatment recommendations. Am J Clin Oncol 2011;34 (04):422-431
28 Toskich B, Vidal LL, Olson MT, et al. Pathologic response of hepatocellular carcinoma treated with yttrium-90 glass microsphere radiation segmentectomy prior to liver transplantation: a validation study. J Vasc Interv Radiol 2021;32(04):518-526.e1

29 Lencioni R, Della Pina C, Bartolozzi C. Percutaneous image-guided radiofrequency ablation in the therapeutic management of hepatocellular carcinoma. Abdom Imaging 2005;30(04):401-408

30 Salem R, Vouche M, Habib A, et al. Reply: To PMID 24691943. Hepatology 2015;61(01):407

31 Seror O, Nault JC, Nahon P, N'Kontchou G, Trinchet JC. Is segmental transarterial yttrium 90 radiation a curative option for solitary hepatocellular carcinoma $\leq 5 \mathrm{~cm}$ ? Hepatology 2015;61(01): 406-407

32 Murthy R, Nunez R, Szklaruk J, et al. Yttrium-90 microsphere therapy for hepatic malignancy: devices, indications, technical considerations, and potential complications. Radiographics 2005; 25(Suppl 1):S41-S55

33 Salem R, Lewandowski RJ, Atassi B, et al. Treatment of unresectable hepatocellular carcinoma with use of $90 \mathrm{Y}$ microspheres (TheraSphere): safety, tumor response, and survival. J Vasc Interv Radiol 2005;16(12):1627-1639

34 Salem R, Thurston KG. Radioembolization with ${ }^{90}$ yttrium microspheres: a state-of-the-art brachytherapy treatment for primary and secondary liver malignancies. Part 1: Technical and methodologic considerations. J Vasc Interv Radiol 2006;17(08): 1251-1278

35 Lawrence TS, Robertson JM, Anscher MS, Jirtle RL, Ensminger WD, Fajardo LF. Hepatic toxicity resulting from cancer treatment. Int J Radiat Oncol Biol Phys 1995;31(05):1237-1248

36 Sangro B, Gil-Alzugaray B, Rodriguez J, et al. Liver disease induced by radioembolization of liver tumors: description and possible risk factors. Cancer 2008;112(07):1538-1546

37 Riaz A, Lewandowski RJ, Kulik LM, et al. Complications following radioembolization with yttrium-90 microspheres: a comprehensive literature review. J Vasc Interv Radiol 2009;20(09):1121-1130 quiz 1131

38 Fernandez-Ros N, Iñarrairaegui M, Paramo JA, et al. Radioembolization of hepatocellular carcinoma activates liver regeneration, induces inflammation and endothelial stress and activates coagulation. Liver Int 2015;35(05):1590-1596

39 Atassi B, Bangash AK, Lewandowski RJ, et al. Biliary sequelae following radioembolization with yttrium-90 microspheres. J Vasc Interv Radiol 2008;19(05):691-697

40 Sangro B, Bilbao JI, Boan J, et al. Radioembolization using 90Yresin microspheres for patients with advanced hepatocellular carcinoma. Int J Radiat Oncol Biol Phys 2006;66(03):792-800

41 Riaz A, Kulik L, Lewandowski RJ, et al. Radiologic-pathologic correlation of hepatocellular carcinoma treated with internal radiation using yttrium-90 microspheres. Hepatology 2009;49 (04):1185-1193

42 Salem R, Mazzaferro V, Sangro B. Yttrium 90 radioembolization for the treatment of hepatocellular carcinoma: biological lessons, current challenges, and clinical perspectives. Hepatology 2013;58 (06):2188-2197

43 O'Connor PJ, Pasik SD, van der Bom IM, Bishay V, Radaelli A, Kim E. Feasibility of yttrium-90 radioembolization dose calculation utilizing intra-procedural open trajectory cone beam CT. Cardiovasc Intervent Radiol 2020;43(02):295-301 